\title{
Particle Induced \\ Electron Emission I
}

With Contributions by

M. Rösler, W. Brauer

and

J. Devooght, J.-C. Dehaes, A. Dubus,

M. Cailler, J.-P. Ganachaud

With 64 Figures

Springer-Verlag

Berlin Heidelberg New York

London Paris Tokyo

Hong Kong Barcelona

Budapest 


\section{Contents}

Theory of Electron Emission from Nearly-Free-Electron Metals by Proton and Electron Bombardment

By $M$. Rösler and W. Brauer (With 42 Figures) $\ldots \ldots \ldots \ldots \ldots \ldots \ldots$

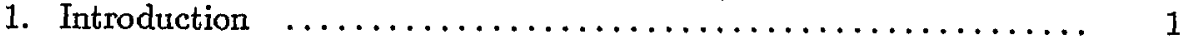

2. Basic Quantities in IIEE and SEE $\ldots \ldots \ldots \ldots \ldots \ldots \ldots \ldots \ldots \ldots$

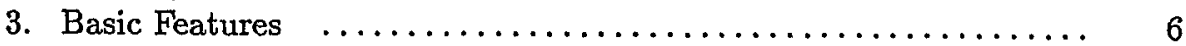

4. Escape of Secondary Electrons $\ldots \ldots \ldots \ldots \ldots \ldots \ldots \ldots \ldots \ldots$

5. Transport Theory of Excited Electrons $\ldots \ldots \ldots \ldots \ldots \ldots \ldots \ldots \ldots$

5.1 Transition Probabilities. Dielectric Function.

Discussion of Different Elementary Processes .......... 10

5.2 General Form of the Transport Equation $\ldots \ldots \ldots \ldots \ldots \ldots 14$

5.3 Homogeneous Excitation $\ldots \ldots \ldots \ldots \ldots \ldots \ldots \ldots \ldots \ldots \ldots \ldots$

6. Scattering Functions and Mean Free Paths $\ldots \ldots \ldots \ldots \ldots \ldots$

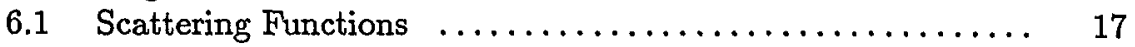

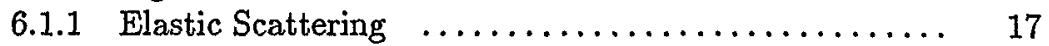

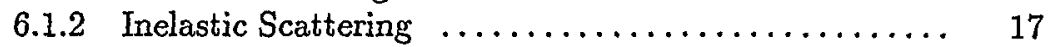

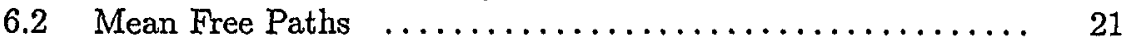

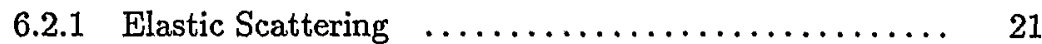

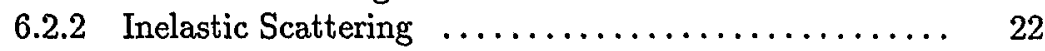

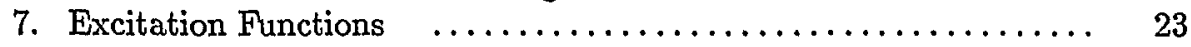

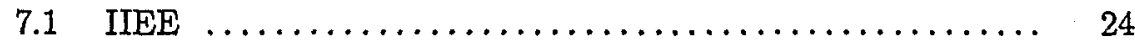

7.1.1 Exicitation of Single Conduction Electrons ....... 25

7.1.2 Excitation by Decay of Plasmons ............. 26

7.1.3 Excitation of Core Electrons ............. 30

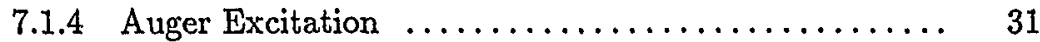

7.1.5 Comparison of Different Excitation Mechanisms ..... 33

7.1.6 Critical Discussion of the Simple Interaction Model . 34

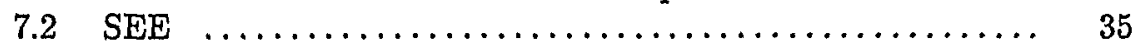

7.2.1 Excitation of Single Conduction Electrons ....... 35

7.2.2 Excitation by Decay of Plasmons ............ 38

7.2.3 Excitation of Core Electrons and Excitation by Auger Processes . ................. 38

7.2.4 Atomic Model of Core Excitations ............ 40

7.2.5 Comparison of Different Excitation Mechanisms ..... 41

8. Solution of the Transport Equation $\ldots \ldots \ldots \ldots \ldots \ldots \ldots \ldots \ldots . \ldots \ldots$

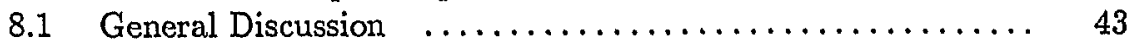

8.2 Monoenergetic Isotropic Excitation $\ldots \ldots \ldots \ldots \ldots \ldots \ldots 4$ 
9. Results for Aluminum and Comparison to Experimental Data ... 46

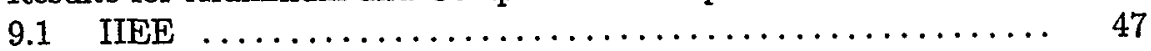

9.1.1 Energy and Angular Distribution $\ldots \ldots \ldots \ldots \ldots \ldots . \ldots 47$

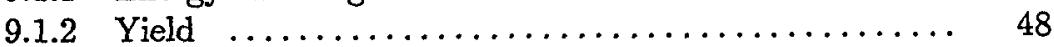

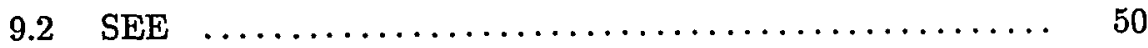

9.2.1 Energy and Angular Distribution $\ldots \ldots \ldots \ldots \ldots \ldots, 50$

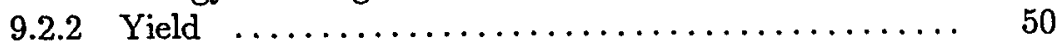

10. The Role of Elastic Scattering $\ldots \ldots \ldots \ldots \ldots \ldots \ldots \ldots \ldots \ldots \ldots$

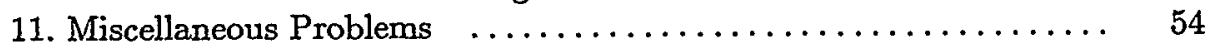

11.1 Results for Thin Films $\ldots \ldots \ldots \ldots \ldots \ldots \ldots \ldots \ldots \ldots \ldots$

11.2 Stopping Power and Electron Yield in IIEE $\ldots \ldots \ldots \ldots \ldots$

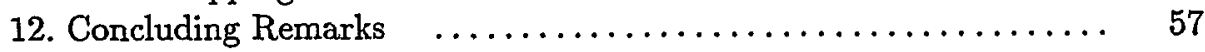

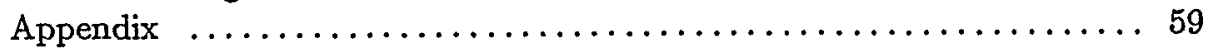

References $\ldots \ldots \ldots \ldots \ldots \ldots \ldots \ldots \ldots \ldots \ldots \ldots \ldots \ldots \ldots \ldots \ldots \ldots \ldots$

Theoretical Description of Secondary Electron Emission Induced

by Electron or Ion Beams Impinging on Solids

By J. Devooght, J.-C. Dehaes, A. Dubus, M. Cailler

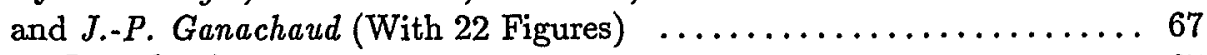

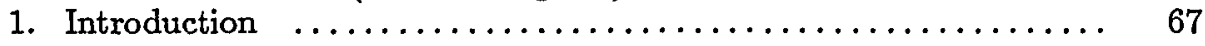

2. Electron Collisions in Solids $\ldots \ldots \ldots \ldots \ldots \ldots \ldots \ldots \ldots \ldots \ldots \ldots$

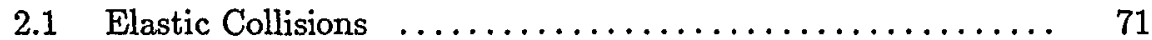

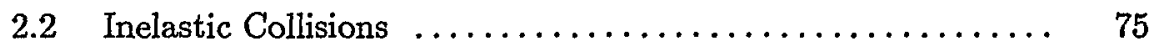

2.2.1 Dielectric Response Functions for NFE Materials .... 75

2.2.2 Bulk and Surface Plasmons .............. 76

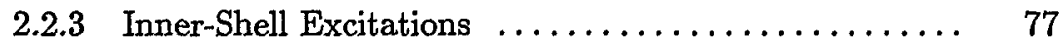

2.3 Extensions to Non-Free-Electron Solids .............. 78

2.3.1 Dielectric Response Functions of a Model Semiconductor or Insulator ......... 78

2.3.2 Use of the Optical Loss Function ............. 78

2.3.3 Extension of the Gryzinski Formulation to the Valence Band .................. 80

2.4 Conclusion $\ldots \ldots \ldots \ldots \ldots \ldots \ldots \ldots \ldots \ldots \ldots \ldots \ldots \ldots$

3. Monte Carlo Simulation Models $\ldots \ldots \ldots \ldots \ldots \ldots \ldots \ldots \ldots \ldots$

3.1 Direct Simulation Scheme $\ldots \ldots \ldots \ldots \ldots \ldots \ldots \ldots \ldots \ldots \ldots . \ldots \ldots$

3.2 Continuous Slowing-Down Scheme $\ldots \ldots \ldots \ldots \ldots . \ldots . \ldots . \ldots$

3.3 Multiple Collision Scheme $\ldots . \ldots \ldots \ldots \ldots \ldots \ldots \ldots . \ldots . \ldots . \ldots$

3.4 Application of Direct MTC Simulation to SEE from Nearly-Free-Electron Materials ............. 84

3.5 Additional Comments About the MTC Simulation Method . 88

4. Transport Models $\ldots \ldots \ldots \ldots \ldots \ldots \ldots \ldots \ldots \ldots \ldots \ldots . \ldots . \ldots . \ldots$

4.1 The Boltzmann Equation $\ldots \ldots \ldots \ldots \ldots \ldots \ldots \ldots \ldots \ldots \ldots \ldots$

4.2 The Age-Diffusion Model $\ldots . \ldots \ldots \ldots \ldots \ldots \ldots \ldots \ldots \ldots . \ldots . \ldots$

4.3 Integral Equations $\ldots \ldots \ldots \ldots \ldots \ldots \ldots \ldots \ldots \ldots \ldots \ldots$

4.3.1 Solution of the Integral Equation in a Semi-Infinite Medium ............. 98 
4.3.2 Transport-Albedo Model $\ldots \ldots \ldots \ldots \ldots \ldots \ldots \ldots, 101$

4.4 Numerical Solution of the Boltzmann Equation ......... 102

4.5 The Model of Schou $\ldots \ldots \ldots \ldots \ldots \ldots \ldots \ldots \ldots .104$

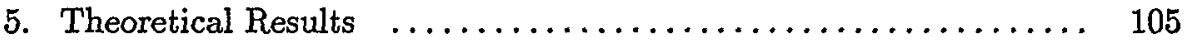

5.1 Electron Emission from a Polycrystalline Al Target ...... 106

5.1.1 SEE Spectrum ......................... 106

5.1.2 Energy-Loss Spectrum .................. 107

5.1.3 Secondary Yield and Backscattering Coefficient ..... 110

5.1.4 Spatial Distribution of Outgoing Electrons ........ 112

5.1.5 Statistical Aspects

of the Electron Emission Phenomenon $\ldots \ldots \ldots \ldots .113$

5.2 SEE from a Polycrystalline Gold Target $\ldots \ldots \ldots \ldots \ldots \ldots . \quad 115$

5.3 Influence of the Primary Electron Transport on the Secondary Electron Yield $\ldots \ldots \ldots \ldots \ldots \ldots \ldots \ldots, 118$

5.4 Proton Induced Electron Emission $\ldots \ldots \ldots \ldots \ldots \ldots \ldots, 120$

5.5 Conclusions $\ldots \ldots \ldots \ldots \ldots \ldots \ldots \ldots \ldots \ldots \ldots \ldots \ldots, 122$

6. Conclusions and Future Prospects $\ldots \ldots \ldots \ldots \ldots \ldots \ldots \ldots \ldots, 123$

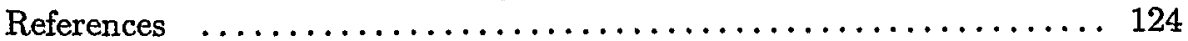

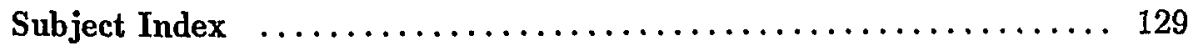

